\title{
Integrability formulation and Bäcklund transformations for gravitational fields with symmetries
}

\author{
F. J. Chinea \\ Departamento de Física Fundamental, U.N.E.D., Madrid, Spain \\ and Departamento de Métodos Matemáticos de la Física, Facultad de Ciencias Físicas, \\ Universidad Complutense de Madrid, Madrid-3, Spain
}

(Received 10 March 1981)

\begin{abstract}
The Ernst equation for gravitational fields with a two-parameter isometry group is formulated as a vanishing-curvature condition on an $\mathrm{SU}(2)$ or $\mathrm{SU}(1,1)$ bundle, both in the elliptic and hyperbolic cases. Bäcklund transformations are introduced as a special case of gauge transformations, and strong Bäcklund transformations are obtained in that context.
\end{abstract}

Gravitational fields with a two-parameter Abelian group of symmetries correspond to situations of great physical interest (e.g., cylindrical gravitational waves, stationary axisymmetric systems). It is well known that the Einstein field equations reduce in that case to the following equations $s^{1,2}$ :

$$
\begin{aligned}
& \omega_{u v}+\frac{\tau_{u}}{2 \tau} \omega_{v}+\frac{\tau_{v}}{2 \tau} \omega_{u}-\left(\omega_{u} \cdot \omega_{v}\right) \omega=0, \\
& \tau_{u v}=0,
\end{aligned}
$$

for the case of cylindrical waves, and

$$
\begin{aligned}
& \omega_{z \bar{z}}+\frac{\tau_{z}}{2 \tau} \omega_{\bar{z}}+\frac{\tau_{\bar{z}}}{2 \tau} \omega_{z}-\left(\omega_{z} \cdot \omega_{\bar{z}}\right) \omega=0, \\
& \tau_{z \bar{z}}=0
\end{aligned}
$$

for stationary axisymmetric systems, where $\tau$ is a real scalar function of the independent variables $u$ and $v$ [ $z$ and $\bar{z}$ in Eq. (2)]. The variables $u$ and $v$ are real, while $z$ is complex, with the bar denoting complex conjugation as usual; $\omega$ is a real vector function depending on $u$ and $v$ ( $z$ and $\bar{z}$ ) in three-dimensional pseudo-Euclidean space with $(++-)$ signature, constrained by the condition

$$
\omega^{2}=-1
$$

Equations (1a) and (2a) are obviously $\mathrm{SO}(2,1)$ invariant. Equation (1a) is equivalent to the Ernst equation ${ }^{3}$

$$
f_{u v}+\frac{\tau_{u}}{2 \tau} f_{v}+\frac{\tau_{v}}{2 \tau} f_{u}-\frac{2 f_{u} f_{v}}{f+\bar{f}}=0
$$

and similarly for Eq. (2a)

$$
f_{\bar{z} \bar{z}}+\frac{\tau_{z}}{2 \tau} f_{\bar{z}}+\frac{\tau_{\bar{z}}}{2 \tau} f_{z}-\frac{2 f_{z} f_{\bar{z}}}{f+\bar{f}}=0,
$$

where $f$ is a complex function.

Equations (4) and (5) are ordinarily derived from the field equations by introducing a certain potential. ${ }^{3}$ They may also be derived algebraically from Eqs. (1a) and $(2 a)$ by simply introducing the parametrization

$$
\omega=\frac{1}{f+\bar{f}}(i[f-\bar{f}], 1-f \bar{f}, 1+f \bar{f}),
$$

which solves the constraint (3). The $\operatorname{SO}(2,1)$ invariance of Eqs. (1a) and (2a) is inherited by Eqs. (4) and (5) in the form of $\operatorname{SL}(2, R)$ invariance under appropriate fractional linear transformations of $f$.

The Ernst equations (4) and (5) may be considered as a vanishing-curvature condition for the connection on a certain bundle with $\mathrm{SU}(2)$ or $\mathrm{SU}(1,1)$ structure group. Such a formulation has proved to be ex- . tremely useful for a variety of equations, such as the sine-Gordon, Korteweg-de Vries, nonlinear Schrödinger equation, etc. It leads to the possibility of using inverse-scattering-transform methods, finding infinite families of conservation laws, and to obtain Bäcklund transformations for those equations. A formulation of this type is available for the vector equations (1a) and (2a). ${ }^{1}$ A moving SU $(1,1)$ invariant trihedral is introduced, and $\omega$ is identified with one of the vectors in the trihedral. The integrability conditions arising from the evolution equations for the trihedral then lead to the desired equation for $\omega$.

We develop in the following a similar formulation for Eqs. (4) and (5), in terms of an SU(2) or SU $(1,1)$ bundle. ${ }^{4}$ Dealing directly with Eqs. (4) and (5) is desirable for the purpose of solution generation and to find conservation laws and Bäcklund transformations.

The bundle connection is given by a pair $(M, N)$ of coordinate-dependent matrices in the corresponding Lie algebra, with the vanishing curvature condition given by

$$
M_{v}-N_{u}+[M, N]=0 .
$$

Equation (6) may be thought of as a consequence of the integrability conditions for the system

$$
A_{u}=M A, A_{v}=N A,
$$

for an appropriate matrix $A$ in the group. 
The $(M, N)$ pair for Eq. (4) is given in the $\mathrm{SU}(2)$ case by

$$
\begin{gathered}
M=\frac{1}{f+\bar{f}}\left(\begin{array}{cc}
\frac{1}{2}(f-\bar{f})_{u} & k f_{u} \\
-\bar{k} \bar{f}_{u} & -\frac{1}{2}(f-\bar{f})_{u}
\end{array}\right), \\
N=\frac{1}{f+\bar{f}}\left(\begin{array}{cc}
\frac{1}{2}(f-\bar{f})_{v} & -\bar{k}^{-1} f_{v} \\
k^{-1} \bar{f}_{v} & -\frac{1}{2}(f-\bar{f})_{v}
\end{array}\right),
\end{gathered}
$$

where $k=(U+\lambda)^{-1 / 2}(V-\lambda)^{1 / 2} e^{i \phi}$, with $U=U(u)$ and $V=V(v)$ being the functions appearing in the solution of (1b): $\tau=U(u)+V(v)$, and $\lambda$ and $\phi$ real constants. The integrability condition (6) for Eq. (7) yields Eq. (4). There is also an $\operatorname{SU}(1,1)$ description of the same equation. The matrices $M$ and $N$ are now given by

$$
\begin{aligned}
& M=\frac{1}{f+\bar{f}}\left(\begin{array}{cc}
\frac{1}{2}(f-\bar{f})_{u} & p f_{u} \\
\bar{p} \bar{f}_{u} & -\frac{1}{2}(f-\bar{f})_{u}
\end{array}\right), \\
& N=\frac{1}{f+\bar{f}}\left(\begin{array}{cc}
\frac{1}{2}(f-\bar{f})_{v} & \bar{p}^{-1} f_{v} \\
p^{-1} \bar{f}_{v} & -\frac{1}{2}(f-\bar{f})_{v}
\end{array}\right),
\end{aligned}
$$

where $p=(-U-\lambda)^{-1 / 2}(V-\lambda)^{1 / 2} e^{i \phi}$ with $U, V, \lambda$, and $\phi$ as before. Finally, the appropriate $(M, N)$ pair for Eq. (5) is the su $(1,1)$ pair

$$
\begin{aligned}
& M=\frac{1}{f+\bar{f}}\left(\begin{array}{cc}
\frac{1}{2}(f-\bar{f})_{x} & \cos \alpha f_{x}+\sin \alpha f_{y} \\
\cos \alpha \bar{f}_{x}+\sin \alpha \bar{f}_{y} & -\frac{1}{2}(f-\bar{f})_{x}
\end{array}\right), \\
& N=\frac{1}{f+\bar{f}}\left(\begin{array}{cc}
\frac{1}{2}(f-\bar{f})_{y} & \cos \alpha f_{y}-\sin \alpha f_{x} \\
\cos \alpha \bar{f}_{y}-\sin \alpha \bar{f}_{x} & -\frac{1}{2}(f-\bar{f})_{y}
\end{array}\right),
\end{aligned}
$$

with $\alpha$ given by $e^{2 i \alpha}=[i \lambda-B(\bar{z})][i \lambda+A(z)]^{-1}$, where $\lambda$ is a real constant and $A(z), B(\bar{z})$ are related to $\tau$ by $\tau(z, \bar{z})=A(z)+B(\bar{z})$, and $z=x+i y, x$ and $y$ real. The condition $M_{y}-N_{x}+[M, N]=0$ is equivalent to Eq. (5).

In this language, Bäcklund transformations may be understood as gauge transformations of the connection $(M, N) .^{5-7}$ This approach has certain computational advantages in the process of finding the analytical expression for the Bäcklund transformations of a given equation. The transformation law for $M$ and $N$ is the usual one for non-Abelian gauge fields (tildes denote transformed quantities)

$$
\tilde{M}=S M S^{-1}+S_{u} S^{-1}, \quad \tilde{N}=S N S^{-1}+S_{v} S^{-1},
$$

where $S$ is a matrix in the group. We now apply this idea to find the explicit form of the Bäcklund transformations for Eq. (4). We work in the SU(2) case given by Eq. (7) [analogous results hold in the SU $(1,1)$ case], and impose the condition that $\tilde{M}$ and $\tilde{N}$ have the same functional form with respect to the transformed solution $\tilde{f}$ as that of $M$ and $N$ with respect to $f$. By assuming $S=S(f, \bar{f}, \tilde{f}, \overline{\tilde{f}} ; u, v)$, the following general form for the Bäcklund transformations of Eq. (4) is obtained from Eq. (10):

$\tilde{f}_{u}=G(f, \bar{f}, \tilde{f}, \overline{\tilde{f}} ; u, v) f_{u}+H(f, \bar{f}, \tilde{f}, \overline{\tilde{f}} ; u, v)$,

$\tilde{f}_{v}=g(f, \bar{f}, \tilde{f}, \overline{\tilde{f}} ; u, v) f_{v}+h(f, \bar{f}, \tilde{f}, \overline{\tilde{f}} ; u, v)$,

where $G, H, g$, and $h$ are functions to be determined below. The algebraic compatibility conditions for the matrix equation (10) force the matrix elements in $S$ to satisfy a system of partial differential equations in the independent variables $f, \bar{f}, \tilde{f}, \overline{\tilde{f}}, u$, and $v$. A simple particular solution is obtained through the Ansatz $S_{f}=S_{\bar{f}}, S_{\tilde{f}}=S_{\bar{f}}$. This results in the following expressions:

$$
\begin{aligned}
& G=(\tilde{f}+\overline{\tilde{f}})(f+\bar{f})^{-1}\left[1-\frac{1}{2} \beta^{2}\left(\kappa+\kappa^{-1}\right)^{2} r-i \beta\left(\kappa+\kappa^{-1}\right) r^{1 / 2}(1+\delta r)^{1 / 2}\right], \\
& H=\beta_{u}(\tilde{f}+\overline{\tilde{f}})\left[\frac{1}{2} \beta\left(1+\kappa^{-2}\right) r+i \kappa^{-1} r^{1 / 2}(1+\delta r)^{1 / 2}\right], \\
& g=(\tilde{f}+\overline{\tilde{f}})(f+\bar{f})^{-1}\left[1-\frac{1}{2} \beta^{2}\left(\kappa+\kappa^{-1}\right)^{2} r+i \beta\left(\kappa+\kappa^{-1}\right) r^{1 / 2}(1+\delta r)^{1 / 2}\right], \\
& h=\beta_{v}(\tilde{f}+\overline{\tilde{f}})\left[\frac{1}{2} \beta\left(1+\kappa^{2}\right) r-i \kappa r^{1 / 2}(1+\delta r)^{1 / 2}\right]
\end{aligned}
$$

where $r=(f+\bar{f})^{-1}(\tilde{f}+\overline{\tilde{f}})^{-1}, \kappa=(U+\lambda)^{-1 / 2}$

$\times(V-\lambda)^{1 / 2}, \beta=(U+\lambda)^{1 / 2}(V-\lambda)^{1 / 2}$, and $\delta=-\frac{1}{4} \beta^{2}\left(\kappa+\kappa^{-1}\right)^{2}$. A constant of integration has been set equal to zero in Eqs. (12) in order to simplify their appearance.

It should be stressed that the transformations given by Eqs. (11) and (12) are strong Bäcklund transformations, in the sense that the integrability conditions for Eqs. (11) imply that both $f$ and $\tilde{f}$ satisfy Eq. (4). Other Ansätze will lead to (in general) different strong Bäcklund transformations for the same equation.
It is well known that Eq. (1a) is intimately related to the field equation for the $\operatorname{SO}(2,1)$-invariant nonlinear $\sigma$ model in two dimensions, to which it in fact reduces when $\tau_{u}=\tau_{v}=0$. The Bäcklund transformations given by Eqs. (11) and (12) possess an appropriate limiting expression in that case. ${ }^{8}$ Simplerlooking Bäcklund transformations may be obtained for the latter, however, by using an Ansatz very specific to that particular case. ${ }^{9}$ Equations similar to Eqs. (11) and (12) hold in the elliptic case given by Eq. (5). 
"Present and permanent address.

${ }^{1}$ D. Maison, Phys. Rev. Lett. 41, 521 (1978); J. Math. Phys. 20, 871 (1979).

2N. Papanicolaou, J. Math. Phys. 20, 2069 (1979).

${ }^{3}$ F. J. Ernst, Phys. Rev. 167, 1175 (1968).

${ }^{4}$ Equation (5) has been written as the integrability condition for a nonmanifestly group-covariant linear system in G Neugebauer, Phys. Lett. 75A, 259 (1980).

${ }^{5} \mathrm{M}$. Crampin, Phys. Lett. 66A, 170 (1978).

${ }^{6}$ R. Sasaki, Nucl. Phys. B154, 343 (1979).

${ }^{7}$ F. J. Chinea, J. Math. Phys. 21, 1588 (1980).

${ }^{8}$ Bäcklund transformations for the Ernst equation (5) in the case $\tau_{z} \neq 0$ have been investigated in B. K. Harrison, Phys. Rev. Lett. 41, 1197 (1978); 41, 1835(E) (1978).

${ }^{9}$ F. J. Chinea, Lett. Math. Phys. (to be published). 